\title{
Recessive multiple epiphyseal dysplasia - Clinical characteristics caused by rare compound heterozygous SLC26A2 genotypes
}

\author{
Mehran Kausar,b $^{\mathrm{a}, \mathrm{b}}$, Riikka E. Mäkitie ${ }^{\mathrm{b}}$, Sanna Toiviainen-Salo ${ }^{\mathrm{c}}$, Jaakko Ignatius ${ }^{\mathrm{d}}$, Mariam Anees ${ }^{\mathrm{a}}$, \\ Outi Mäkitie $\mathrm{b}, \mathrm{e}, \mathrm{g}, *^{\mathrm{s}, *}$ \\ ${ }^{a}$ Department of Biochemistry, Quaid-i-Azam University, Islamabad, Pakistan \\ ${ }^{\mathrm{b}}$ Folkhälsan Institute of Genetics and University of Helsinki, Helsinki, Finland \\ ${ }^{\mathrm{c}}$ Department of Pediatric Radiology, HUS Medical Imaging Centre, University of Helsinki and Helsinki University Hospital, Helsinki, Finland \\ ${ }^{\mathrm{d}}$ Department of Clinical Genetics, University of Turku and Turku University Hospital, Turku, Finland \\ ${ }^{\mathrm{e}}$ Children's Hospital, University of Helsinki and Helsinki University Hospital, Helsinki, Finland \\ ${ }^{\mathrm{f}}$ Department of Molecular Medicine and Surgery and Center for Molecular Medicine, Karolinska Institutet, Stockholm, Sweden \\ ${ }^{\mathrm{g}}$ Department of Clinical Genetics, Karolinska University Hospital, Stockholm, Sweden
}

\section{A R T I C L E I N F O}

\section{Keywords:}

rMED

Chondrodysplasia

SLC26A2

Double-layer patella

Robin sequence

\begin{abstract}
A B S T R A C T
Pathogenic sequence variants in the solute carrier family 26 member 2 (SLC26A2) gene result in lethal (achondrogenesis Ib and atelosteogenesis II) and non-lethal (diastrophic dysplasia and recessive multiple epiphyseal dysplasia, rMED) chondrodysplasias. We report on two new patients with rMED and very rare compound heterozygous mutation combinations in non-consanguineous families. Patient I presented in childhood with waddling gait and joint stiffness. Radiographs showed epiphyseal changes, bilateral coxa plana-deformity and knee valgus deformity, for which he underwent surgeries. At present 33 years his height is $165 \mathrm{~cm}$. Patient II presented with cleft palate, small jaw, short limbs, underdeveloped thumbs and on radiographs, cervical kyphosis with an underdeveloped C4. He also developed severe scoliosis but has grown at -2.9 SD curve. Molecular analysis revealed that patient I is heterozygous for two known pathogenic variants in $S L C 26 A 2$, a splice site variant c. $-26+2 \mathrm{~T}>\mathrm{C}$ and a missense variant c.1957T $>$ A (p.Cys653Ser), while patient II is compound heterozygous for missense variants c.835C > T (p.Arg279Trp) and c.1535C > A (p.Thr512Lys). These patients further elucidate the variability of the phenotypic and genetic presentations of rMED.
\end{abstract}

\section{Introduction}

Autosomal recessive multiple epiphyseal dysplasia (rMED; OMIM \#226900) is a rare and relatively mild form of chondrodysplasia caused by mutations in SLC26A2 (OMIM 606718) (Superti-Furga et al., 1999) (Mäkitie et al., 2003). Solute carrier family 26 member 2 (SLC26A2) is a transmembrane sulfate carrier protein that partakes in sulfation of proteoglycans by transporting sulfate into the cartilage matrix. Mutations in SLC26A2 result in inappropriately constructed cartilage matrix; undersulfated proteoglycans hamper bone development especially at long bones epiphyses, large joints, and metacarpals and metatarsals. To date, 51 different SLC26A2 mutations have been reported to associate with four chondrodysplasias: achondrogenesis Ib, atelosteogenesis II, diastrophic dysplasia (DTD) and rMED. The most frequent SLC26A2 mutation in the Finnish population, where DTD is exceptionally common, is c. $-26+2 \mathrm{~T}>\mathrm{C}$, also known as the Finnish founder mutation (Hastbacka et al., 1999). In other populations, the most prevalent mutation is c.862C > T (p.Arg279Trp) (Syvanen et al., 2013).

The phenotype of rMED varies according to the type of SLC26A2 mutation (Czarny-Ratajczak et al., 2010). The main clinical features include normal to mildly short stature, joint contractures, clubfeet, shortening of limbs and waddling gait; radiographs show epiphyseal dysplasia (Bonafe et al., 2008) (Mäkitie et al., 2003). Recent reports suggest a wider spectrum of clinical manifestations, including cleft palate and auricular deformities (Mäkitie et al., 2015).

Here we present two patients, a 33-year-old and a 5-year-old male, with rMED due to very rare compound heterozygosity for SLC26A2 mutations. Both have relatively normal growth but show also manifestations that are more typical for DTD rather than rMED, further evidencing a clinical overlap of these conditions.

\footnotetext{
${ }^{*}$ Corresponding author. Folkhälsan Institute of Genetics, P.O.Box 63, FIN-00014, University of Helsinki, Finland.

E-mail address: outi.makitie@helsinki.fi (O. Mäkitie).
} 


\section{Clinical report}

This retrospective study is part of an ongoing research program on epidemiological, clinical and genetic characteristics of skeletal dysplasia in Finland, carried out at Children's Hospital, Helsinki University Hospital, Finland. The protocol was approved by the Institutional Research Ethics Committee. The two patients had been followed at various clinics at Helsinki University Hospital (Patient I) and Turku University Hospital (Patient II) and were referred to the Clinical Genetics Department, Helsinki University Hospital in 2017 for further evaluation.

The patients' data from hospital records were reviewed for clinical presentation, disease progression, anthropometry and radiographic skeletal manifestations. Growth data were compared with Finnish growth standards. Genetic studies were performed from peripheral blood genomic DNA; for patient I, a gene panel including SLC26A2 was performed at Blueprint Genetics (Helsinki, Finland) and the identified SLC26A2 variants were confirmed by Sanger sequencing. For patient II, SLC26A2 was screened at HUSLAB (Helsinki, Finland) for two Finnish major mutations and subsequently for all exons.

\subsection{Patient I}

This presently 33-year-old man was born at full term to non-consanguineous parents who were healthy and normal in height. The birth measurements were normal: length $49 \mathrm{~cm}(-1.2$ standard deviation units, SDS), weight $3845 \mathrm{~g}(+0.3 \mathrm{SDS})$, and head circumference $36.5 \mathrm{~cm}(+0.8 \mathrm{SDS})$. He was treated for bilateral hip dysplasia after birth. He walked at 12 months but due to abnormal gait, he underwent clinical investigations at 2 years. He was noticed to have diffuse skeletal epiphyseal changes that were most prominent in his lower limbs; he had bilateral coxa plana deformity and his knees were in valgus position. Despite normal spine, he was diagnosed with spondylo-epiphyseal dysplasia; no molecular diagnosis was performed. During childhood he underwent surgeries to correct his knee valgus deformity and hip and knee surgeries are presently considered because of joint pain. Other joints and the spine are less severely affected and only exhibit mild stiffness and restricted range of motion. His hands and feet are slightly wide and stubby and both fourth metacarpals and metatarsals are short. There is no clubfoot deformity. He has no apparent facial dysmorphias, extra-skeletal abnormalities or dental problems, and has normal vision, hearing and intelligence. He has no scoliosis and has reached an adult height of $165 \mathrm{~cm}(-2.5 \mathrm{SDS})$.

Radiographs between 6 and 33 years of age were reviewed (Fig. 1). At 6 years, he had flat crescent-shaped femoral epiphyses, abnormally broad and short femoral necks, and a dysplastic right acetabulum. Epiphyses and metaphyses of the knees were abnormal. The double layer patellae were laterally displaced. All metacarpals, especially the fourth, were short. Epiphyses of both radius and ulnae were slightly flat. Lumbar vertebrae were of normal height and shape. At 18 years he had developed bilateral severe hip arthrosis and the right femoral head was cranially dislocated. At 33 years the patient had marked arthrosis in both hips and knees.

\subsection{Patient II}

This presently 5 -year-old boy was born by elective Cesarian section at $38+6$ gestational weeks from breech position measuring $47 \mathrm{~cm}$ $(-1.9$ SDS $)$ and $3230 \mathrm{~g}(-0.8 \mathrm{SDS})$; his head circumference was $35.5 \mathrm{~cm}(+0.3 \mathrm{SDS})$. The parents were non-consanguineous, healthy and normal in height. Routine ultrasounds during pregnancy showed normal morphology and growth. Immediately after birth, he was noticed to have a wide cleft palate exposing inferior nasal structures, small jaw suggesting Pierre Robin-sequence, overall short limbs and length, spontaneous palmar ulnar deviation and underdeveloped thumbs (Fig. 2). Ultrasound showed femoral heads to be abnormal in shape.
Cervical radiographs indicated kyphosis with an underdeveloped C4. Cleft palate was closed at 1 year and his jaw has grown spontaneously. He developed severe progressive scoliosis during the second year and was treated with casting and a corset until present age.

Presently, at 5 years, his height is $98 \mathrm{~cm}(-3.0$ SDS) and weight $19 \mathrm{~kg}$ (0 SDS) (Fig. 2). He has developed significant valgus deformity at knees. His ankles are stiff but there is no clubfoot deformity. Joint stiffness is also present in upper limbs and he has contractures in both third fingers. Both earlobes are slightly thick and low set. His head circumference is normal ( -0.5 SDS).

On radiographs, at age 2 days, both upper and lower limbs showed broad metaphyses and elbow contractures (Fig. 3). Cervical kyphosis with underdeveloped fourth cervical vertebrae was present at 3 months but was mostly resolved spontaneously by 5 years. Thoracic and lumbar vertebrae were of normal shape but there was significant scoliosis. Lower extremities at 5 years showed bilateral coxa vara with abnormally broad and short femoral necks and flat epiphyses, and both knees were in valgus position. Knee MRI demonstrated bilateral laterally displaced double layer patellae.

\subsection{Genetic findings}

Patient I had been previously diagnosed as having spondyloepiphyseal dysplasia but as the characteristics were atypical for COL2A1related skeletal dysplasia, a bone dysplasia gene panel was performed at Blueprint Genetics, which indicated heterozygosity for two known SLC26A2 pathogenic variants: c. $-26+2 \mathrm{~T}>\mathrm{C}$ (rs386833492) and c.1957T > A (p.Cys653Ser, rs104893924) (Fig. 4). The former is a well-known disease-causing splice donor site mutation and recognized as the Finnish founder mutation for DTD (Hastbacka et al., 1999). The latter is a missense variant with pathogenic predictions by SiFT and PolyPhen and a rather low allele frequency in databases. Homozygous p.Cys653Ser has previously been described in relatively mild rMED (Mäkitie et al., 2003). The parents were healthy and were not available for genetic testing.

Genetic testing for Patient II for suspected SLC26A2-related disease was performed in the first days postpartum at HUSLAB (Helsinki, Finland) for the Finnish major mutations and subsequently for the whole SLC26A2 gene. The patient was found to harbor two heterozygous missense mutations in SLC26A2 (NM_000112.3): the common variant c.835C > T (p.Arg279Trp, rs104893915; paternally inherited) and a rare mutation c.1535C > A (p.Thr512Lys, rs121908078; maternally inherited), which has only been found in the Finnish population (submitted to LOVD, accession number 00181232) (Fig. 4). His clinical characteristics, particularly his mildly short stature, and genetic findings were not typical for DTD and his diagnosis was regarded as rMED or variant of DTD.

\section{Discussion}

We describe two patients with unusual combinations of SLC26A2 mutations leading to an rMED phenotype. These patients elucidate the genetic and clinical variability of this entity and further underscore the overlap between rMED and DTD. Further, as seen in Patient II, some of these manifestations may be severe and require prompt treatment (e.g. severe cervical kyphosis, early-childhood onset scoliosis) and therefore careful follow up of these patients is indicated.

The SLC26A2 gene comprises three exons and encodes a 739 amino acid transmembrane sulfate carrier protein (SLC26A2) (Hastbacka et al., 1994). Pathogenic sequence variants in SLC26A2 cause various forms of recessive chondrodysplasias with mild to severe phenotypes including achondrogenesis type 1B (OMIM 600972), atelosteogenesis type II (OMIM 256050), diastrophic dysplasia (OMIM 222600) and recessive MED (OMIM 226900). There are 51 SLC26A2 mutations listed in the Human Gene Mutation Database professional (www.hgmd.cf.ac. uk): 29 are missense mutations, three nonsense mutations, two splice 


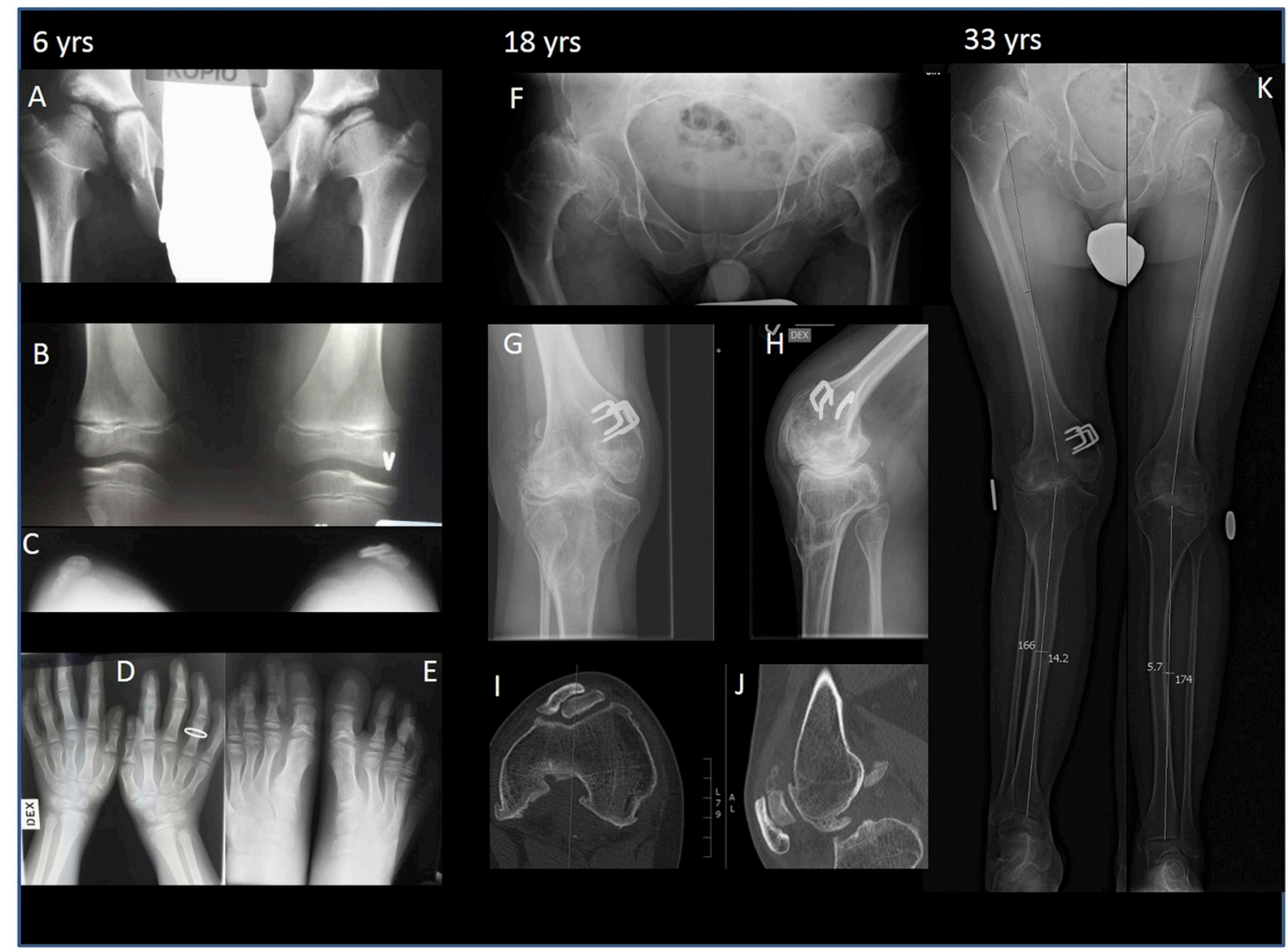

Fig. 1. Radiographs of Patient 1 from age 6 to 33 years.

A. Hip X-rays at 6 years showed bilaterally flattened, crescent-shaped femoral epiphyses, broad and short femoral necks, and dysplastic right acetabulum.

B. At age 6 years, epiphyses of the knees were slightly flat, and

C. patient had laterally displaced double layer patellae.

D. Hand X-ray of both hands showed short, "stubby" metacarpals, fourth metacarpal bones being particularly short. Epiphyses of ulna and radius were slightly flat, and there was ulnar deviation in the left wrist.

E. All metatarsals were also short and "stubby" with flat distal epiphyses, the fourth and the fifth metatarsals being particularly short. The proximal phalanges of the both first toes had cone epiphyses.

F. Pelvis X-ray at age 18 years demonstrated cranially dislocated right femoral head and severe hip arthrosis on both hips.

G-H. X-rays of the right knee with severe arthrosis (metal hooks from medial epiphyseodesis operation)

I-J. CT of the left knee with double layer patella and knee arthrosis

$\mathrm{K}$. In X-ray of the lower extremities at age 33 years there was marked arthrosis in the both hips and knees. Right hip was cranially dislocated, and right knee was in valgus position.

site mutations and 16 frameshift mutations. ClinVar (www.ncbi.nlm. nih.gov/clinvar) reports 58 SLC26A2 variants that are classified as pathogenic or likely pathogenic. Several of these mutations have been reported as causing rMED (Table 1), the most common genotype in rMED being homozygosity for c.835C $>\mathrm{T}$ (p.Arg279Trp) (Bonafe et al., 2002; Barbosa et al., 2011). The phenotypic severity can be correlated with the residual activity of the sulfate transporters resulting from different mutant alleles (Rossi and Superti-Furga, 2001; Syvanen et al., 2013).

A previous report indicated that patients with rMED may have similar cervical kyphosis as seen in DTD (Mäkitie et al., 2015). This was also seen in our Patient II. Here we further report the presence of severe
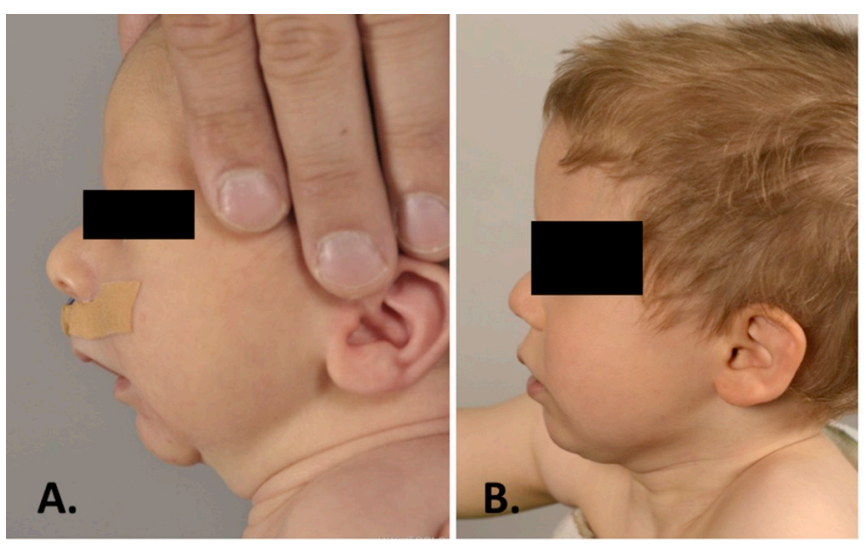

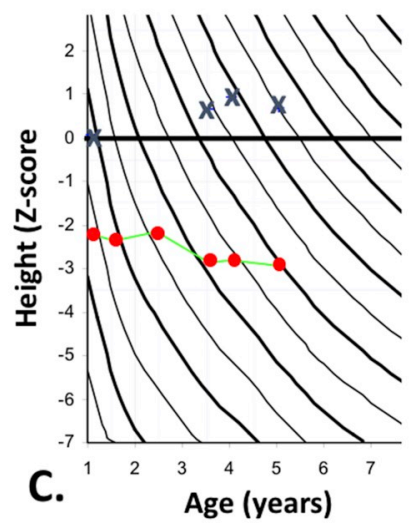

Fig. 2. Clinical features of Patient 2.

Patient 2 at 2 weeks (A.) and at 3 years (B.). The small jaw has grown spontaneously. The growth curve (dots connected by line) shows relative height deviating from $-2.1 \mathrm{SD}$ to $-2.9 \mathrm{SD}$ at the same time when scoliosis and knee valgus deformity have deteriorated. Height-adjusted weight (crosses) has remained normal. 


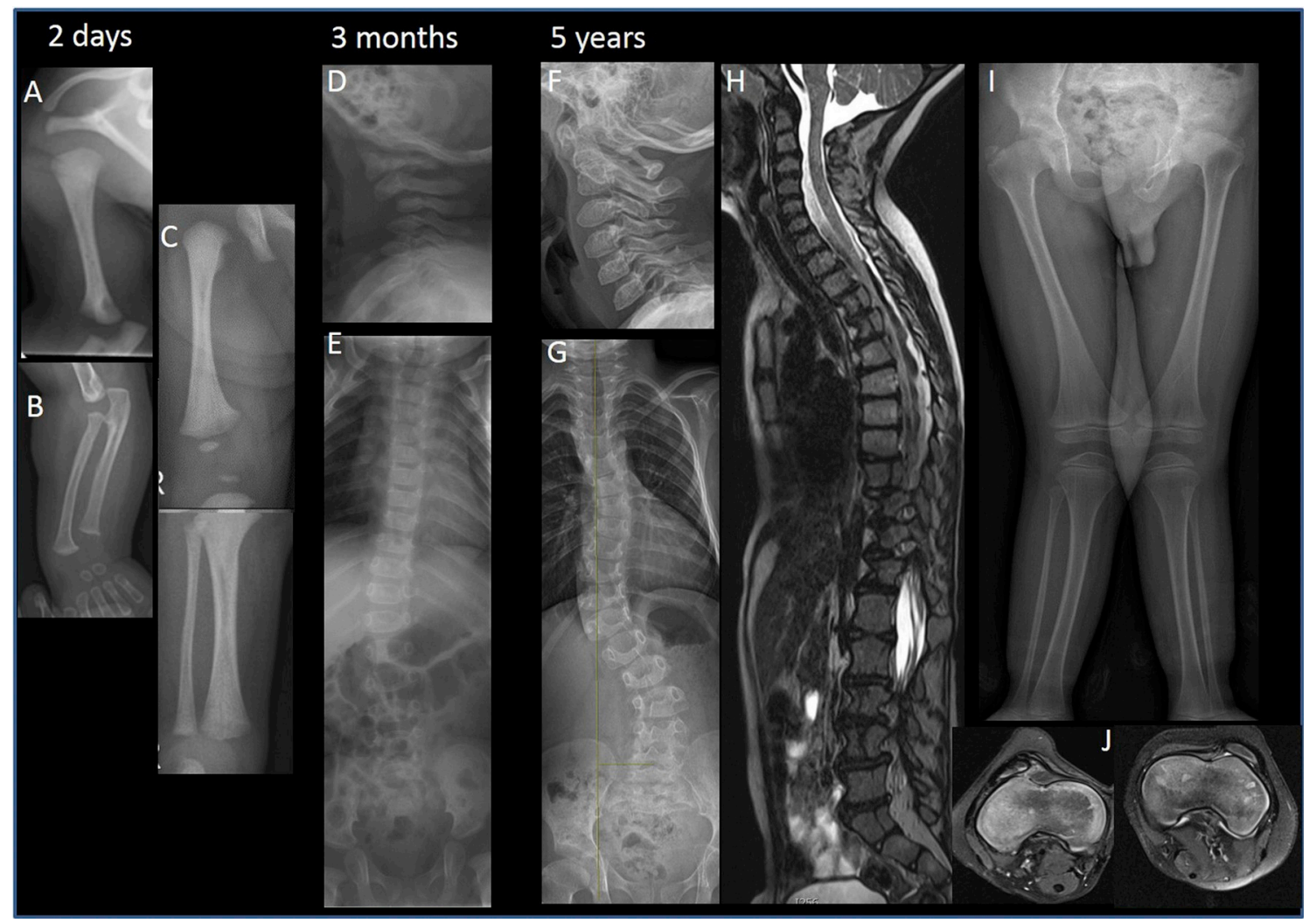

Fig. 3. Radiographs of Patient 2 from age 2 days to 5 years.

A-B. X-rays of the right arm and C. right leg at age 2 days with broad metaphyses of the tubular bones.

D-E: In spinal X-ray at 3 months, cervical kyphosis with abnormal, underdeveloped fourth cervical vertebra was evident. Thoracic and lumbar vertebrae had normal shape, there was a slight curvature at the thoraco-lumbar level.

F-H. At 5 years of age, cervical kyphosis had resolved spontaneously, but patient had developed scoliosis (F. neck X-ray G. spine X-ray, H. spine MRI).

I: X-ray of the lower extremities at age 5 years with bilateral coxa vara with abnormally broad and short femoral necks and flat epiphyses, and both knees in valgus position.

J: In MRI (axial fat-saturated PD TSE sequence) there were laterally displaced double layer patellae in both knees.

scoliosis in a patient with rMED. Furthermore, the patient also had a very small jaw with cleft palate, reminiscent of Pierre Robin-sequence, which was also considered as his diagnosis. In 2013, Zechi-Ceide et al. reported on two siblings with compound heterozygosity for SCL26A2 mutations p.Arg279Trp and the Finnish founder mutation (c.$26+2 \mathrm{~T}>\mathrm{C}$ ) and Robin sequence, and a phenotype intermediate between DTD and rMED (Zechi-Ceide et al., 2013). Interestingly, in our patient the disproportionately small jaw has normalized by age 5 years.

Our Patient I had received a diagnosis of spondylo-epiphyseal dysplasia, which is an autosomal dominant disease with a $50 \%$ risk of recurrence in the offspring. Careful clinical and radiographic review, combined with genetic testing, confirmed the correct diagnosis to be rMED, with remarkably lower inheritance risk even in the Finnish population with higher than normal carrier frequency for SLC26A2 mutations.

In summary, our report further elucidates the phenotypic and genotypic variability of rMED. The severe manifestations in one of our patients highlight the need for careful clinical follow up of patients with

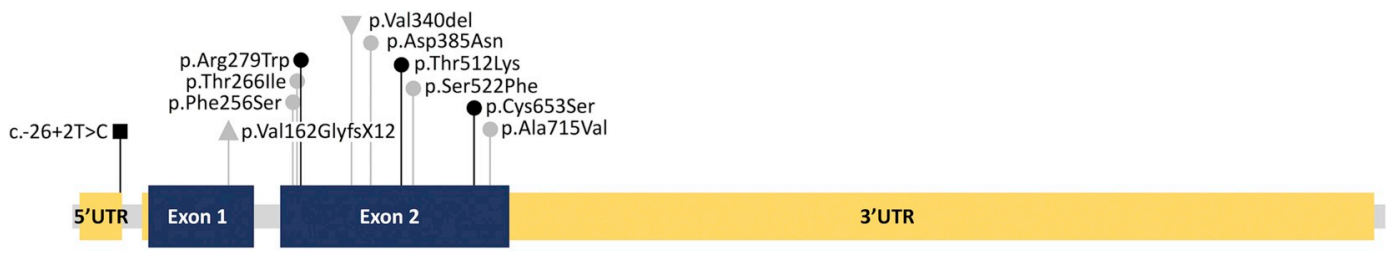

$\begin{array}{ll}\text { UTR regions } & \text { Deletion } \\ \text { Exonic regions } & \text { Frameshift } \\ \text { Intronic regions } & \text { Missense } \\ & \end{array}$

Fig. 4. Schematic presentation of rMED-causing SLC26A2 mutations reported in literature.

The mutation types is indicated with different symbols. The SLC26A2 mutations described in this article are marked in black and previously reported mutations in gray. UTR = untranslated region. 
Table 1

List of known mutations in SLC26A2 causing rMED.

\begin{tabular}{|c|c|c|c|c|c|}
\hline Allele A & & Allele B & & Phenotype & Reported by \\
\hline c. $862 \mathrm{C}>\mathrm{T}$ & p.Arg279Trp & c. $862 \mathrm{C}>\mathrm{T}$ & p.Arg279Trp & rMED & $\begin{array}{l}\text { Superti-Furga et al., [1999], Huber et al., [2001], Czarny-Ratajczak et al., } \\
\text { [2010], Ballhausen et al., [2003] }\end{array}$ \\
\hline c. $1984 \mathrm{~T}>\mathrm{A}$ & p.Cys653Ser & c. $1984 \mathrm{~T}>\mathrm{A}$ & p.Cys653Ser & rMED & Mäkitie et al., [2003], Hinrichs et al., [2010] \\
\hline c. $1984 \mathrm{~T}>\mathrm{A}$ & p.Cys653Ser & c. $2172 \mathrm{C}>\mathrm{T}$ & p.Ala715Val & rMED & $\begin{array}{l}\text { Rossi and Superti-Furga [2001], Ballhausen et al., [2003], Jackson et al., } \\
\text { [2012] }\end{array}$ \\
\hline c. $-26+2 \mathrm{~T}>\mathrm{C}$ & IVS1 $+2 \mathrm{~T}$ & c.794T > C & p.Phe256Ser & rMED & Hästbacka et al., [1999], Jackson et al., [2012] \\
\hline c. $-26+2 \mathrm{~T}>\mathrm{C}$ & $\mathrm{IVS} 1+2 \mathrm{~T}$ & c. $1984 \mathrm{~T}>\mathrm{A}$ & p.Cys653Ser & rMED & Hästbacka et al., [1999], Ballhausen et al., [2003], Jackson et al., [2012] \\
\hline c. $862 \mathrm{C}>\mathrm{T}$ & p.Arg279Trp & c. $1562 \mathrm{C}>\mathrm{A}$ & p.Thr512Lys & rMED & Syvanen et al., [2013] \\
\hline c. $-26+2 \mathrm{~T}>\mathrm{C}$ & IVS1 $+2 \mathrm{~T}$ & c. $862 \mathrm{C}>\mathrm{T}$ & p.Arg279Trp & rMED/DTD & Ballhausen et al., [2003] \\
\hline c. $485 \_486$ delTG & p.Val162GlyfsX12 & c. $1153 \mathrm{G}>\mathrm{A}$ & p.Asp385Asn & rMED & Cho et al., [2010] \\
\hline c.797C > T & p.Thr266Ile & c.1018-1020del & p.Val340del & $\begin{array}{l}\text { rMED/ Desbuquios } \\
\text { dysplasia }\end{array}$ & Miyake et al., [2008] \\
\hline c. $835 \mathrm{C}>\mathrm{T}$ & p.Arg279Trp & c. $1565 \mathrm{C}>\mathrm{T}$ & p.Ser522Phe & rMED & Barreda-Bonis et al., [2018] \\
\hline c. $-26+2 \mathrm{~T}>\mathrm{C}$ & IVS1 + 2T & c. $1957^{\mathrm{a}} \mathrm{T}>\mathrm{A}$ & p.Cys653Ser & rMED & This report (Patient $\mathrm{I}$ ) \\
\hline c. $835^{\mathrm{a}} \mathrm{C}>\mathrm{T}$ & p.Arg279Trp & c. $1535^{\mathrm{a}} \mathrm{C}>\mathrm{A}$ & p.Thr512Lys & rMED & This report (Patient II) \\
\hline
\end{tabular}

${ }^{\text {a }}$ NM_000112.3 (Reference sequence differ from older version).

rMED. Furthermore, we emphasize the importance of correct molecular diagnosis in skeletal dysplasias as this has a major impact on genetic counseling.

\section{Funding}

This study was financially supported by the Academy of Finland, Sigrid Jusélius Foundation, Foundation for Pediatric Research, Folkhälsan Research Foundation, Swedish Research Council, the Novo Nordisk Foundation, Stockholm County Council, University of Helsinki through the Doctoral Programme in Clinical Research, and Helsinki University Hospital research funds.

\section{Conflicts of interest}

All authors declare no conflicts of interest.

\section{Appendix A. Supplementary data}

Supplementary data to this article can be found online at https:// doi.org/10.1016/j.ejmg.2018.11.007.

\section{References}

Ballhausen, D., Bonafe, L., Terhal, P., Unger, S.L., Bellus, G., Classen, M., Hamel, B.C., Spranger, J., Zabel, B., Cohn, D.H., Cole, W.G., Hecht, J.T., Superti-Furga, A., 2003. Recessive multiple epiphyseal dysplasia (rMED): phenotype delineation in eighteen homozygotes for DTDST mutation R279W. J. Med. Genet. 40, 65-71.

Barbosa, M., Sousa, A.B., Medeira, A., Lourenco, T., Saraiva, J., Pinto-Basto, J., Soares, G., Fortuna, A.M., Superti-Furga, A., Mittaz, L., Reis-Lima, M., Bonafe, L., 2011. Clinical and molecular characterization of Diastrophic Dysplasia in the Portuguese population. Clin. Genet. 80, 550-557.

Bonafe, L., Blanton, S.H., Scott, A., Broussard, S., Wise, C.A., Superti-Furga, A., Hecht, J.T., 2002. DTDST mutations are not a frequent cause of idiopathic talipes equinovarus (club foot). J. Med. Genet. 39 e20.

Bonafe, L., Hastbacka, J., de la Chapelle, A., Campos-Xavier, A.B., Chiesa, C., Forlino, A., Superti-Furga, A., Rossi, A., 2008. A novel mutation in the sulfate transporter gene SLC26A2 (DTDST) specific to the Finnish population causes de la Chapelle dysplasia. J. Med. Genet. 45, 827-831.

Cho, T.J., Kim, O.H., Lee, H.R., Shin, S.J., Yoo, W.J., Park, W.Y., Park, S.S., Cho, S.I., Choi, I.H., 2010. Autosomal recessive multiple epiphyseal dysplasia in a Korean girl caused by novel compound heterozygous mutations in the DTDST (SLC26A2) gene. J. Kor.
Med. Sci. 25, 1105-1108.

Czarny-Ratajczak, M., Bieganski, T., Rogala, P., Glowacki, M., Trzeciak, T., Kozlowski, K., 2010. New intermediate phenotype between MED and DD caused by compound heterozygous mutations in the DTDST gene. Am. J. Med. Genet. 152A, 3036-3042.

Hastbacka, J., Kerrebrock, A., Mokkala, K., Clines, G., Lovett, M., Kaitila, I., de la Chapelle, A., Lander, E.S., 1999. Identification of the Finnish founder mutation for diastrophic dysplasia (DTD). Eur. J. Hum. Genet. 7, 664-670.

Hastbacka, J., de la Chapelle, A., Mahtani, M.M., Clines, G., Reeve-Daly, M.P., Daly, M., Hamilton, B.A., Kusumi, K., Trivedi, B., Weaver, A., et al., 1994. The diastrophic dysplasia gene encodes a novel sulfate transporter: positional cloning by fine-structure linkage disequilibrium mapping. Cell 78, 1073-1087.

Hinrichs, T., Superti-Furga, A., Scheiderer, W.D., Bonafe, L., Brenner, R.E., Mattes, T., 2010. Recessive multiple epiphyseal dysplasia (rMED) with homozygosity for C653S mutation in the DTDST gene-phenotype, molecular diagnosis and surgical treatment of habitual dislocation of multilayered patella: case report. BMC Musculoskelet. Disord. 11, 110.

Huber, C., Odent, S., Rumeur, S., Padovani, P., Penet, C., Cormier-Daire, V., Munnich, A., Le Merrer, M., 2001. Sulphate transporter gene mutations in apparently isolated club foot. J. Med. Genet. 38, 191-203.

Jackson, G.C., Mittaz-Crettol, L., Taylor, J.A., Mortier, G.R., Spranger, J., Zabel, B., Le Merrer, M., Cormier-Daire, V., Hall, C.M., Offiah, A., Wright, M.J., Savarirayan, R., Nishimura, G., Ramsden, S.C., Elles, R., Bonafe, L., Superti-Furga, A., Unger, S., Zankl, A., Briggs, M.D., 2012. Pseudoachondroplasia and multiple epiphyseal dysplasia: a 7-year comprehensive analysis of the known disease genes identify novel and recurrent mutations and provides an accurate assessment of their relative contribution. Hum. Mutat. 33, 144-157.

Makitie, O., Geiberger, S., Horemuzova, E., Hagenas, L., Mostrom, E., Nordenskjold, M., Grigelioniene, G., Nordgren, A., 2015. SLC26A2 disease spectrum in Sweden - high frequency of recessive multiple epiphyseal dysplasia (rMED). Clin. Genet. 87, 273-278.

Mäkitie, O., Savarirayan, R., Bonafe, L., Robertson, S., Susic, M., Superti-Furga, A., Cole, W.G., 2003. Autosomal recessive multiple epiphyseal dysplasia with homozygosity for C653S in the DTDST gene: double-layer patella as a reliable sign. Am. J. Med. Genet. 122A, 187-192.

Rossi, A., Superti-Furga, A., 2001. Mutations in the diastrophic dysplasia sulfate transporter (DTDST) gene (SLC26A2): 22 novel mutations, mutation review, associated skeletal phenotypes, and diagnostic relevance. Hum. Mutat. 17, 159-171.

Superti-Furga, A., Neumann, L., Riebel, T., Eich, G., Steinmann, B., Spranger, J., Kunze, J., 1999. Recessively inherited multiple epiphyseal dysplasia with normal stature, club foot, and double layered patella caused by a DTDST mutation. J. Med. Genet. 36, 621-624.

Syvanen, J., Helenius, I., Hero, M., Makitie, O., Ignatius, J., 2013. Recessive MED with auricular swelling due to compound heterozygosity Arg279Tpr/Thr512Lys in the SLC26A2 gene. Am. J. Med. Genet. 161A, 1491-1494.

Zechi-Ceide, R.M., Moura, P.P., Raskin, S., Richieri-Costa, A., Guion-Almeida, M.L., 2013. A compound heterozygote SLC26A2 mutation resulting in robin sequence, mild limbs shortness, accelerated carpal ossification, and multiple epiphysial dysplasia in two Brazilian sisters. A new intermediate phenotype between diastrophic dysplasia and recessive multiple epiphyseal dysplasia. Am. J. Med. Genet. 161A, 2088-2094. 